\title{
Performance of a Simplified Wall Motion Score Index Method for Noncardiologists to Assess Left Ventricular Ejection Fraction
}

\author{
Réal Lebeau, ${ }^{1}$ Brian J. Potter, ${ }^{2}$ Georgetta Sas, ${ }^{1}$ Sherif Moustafa, ${ }^{1}$ Maria Di Lorenzo, ${ }^{1}$ \\ Vicky Soulieres, ${ }^{1}$ Yannick Beaulieu, ${ }^{1}$ Claude Sauvé, ${ }^{1}$ Robert Amyot, ${ }^{1}$ and Karim Serri ${ }^{1}$ \\ ${ }^{1}$ Hôpital du Sacré-Coeur de Montréal, Université de Montréal, 5400 Boulevard Gouin Ouest, Montréal, QC, Canada H4J 1C5 \\ ${ }^{2}$ Centre Hospitalier de l'Université de Montréal, Université de Montréal, Montréal, QC, Canada H2W 1T8
}

Correspondence should be addressed to Réal Lebeau, real.lebeau@yahoo.ca

Received 23 July 2011; Accepted 6 September 2011

Academic Editors: M. Pocar, W. Ryotaro, and E. E. Wang

Copyright ( 2012 Réal Lebeau et al. This is an open access article distributed under the Creative Commons Attribution License, which permits unrestricted use, distribution, and reproduction in any medium, provided the original work is properly cited.

\begin{abstract}
Backgrounds. For emergency physicians performing a goal-directed transthoracic echocardiogram (TTE), a reliable estimate of LVEF must be obtained rapidly. We compared rapid LVEF estimates obtained from short axis sections to those obtained from apical sections using two methods of evaluation. Methods. The TTE's of 6 patients were interpreted by 16 echo-proficient readers (PRO group) and 105 novice readers (NOV group). LVEF was assessed by each group. The strategies consisted of either a global visual estimation (VIS) of LVEF or semiquantitative (SQ) methods. Results. Using RNV and BIP as a reference standard, NOV readers performed better with the SQ method than global visual estimation. For NOV readers, best agreement was achieved with the 234C sequence in low LVEF situations, but with the BMA series in normal LVEF settings. Neither series of views was better than the other in the setting of mild LVEF depression. Conclusion. Semi-quantitative method was superior to global visual estimation of LVEF in NOV group in most of the LVEF ranges.
\end{abstract}

\section{Introduction}

Emergency and intensive care physicians and other noncardiologists are frequently called upon to manage critically ill patients with various pathologies that impact cardiac function, including pericardial effusion, pulmonary embolism, sepsis, and hypovolemic shock. Accurate and timely assessment of global cardiac function can be critical in subsequent diagnostic assessment and medical decision making. As such, bedside echocardiography is becoming a tool of increasing clinical relevance in a variety of cardiac diseases (coronary artery disease and congestive heart failure) from the emergency department to the intensive care unit [1]. Although algorithm-derived computations of the left ventricular ejection fraction (LVEF), such as the Simpson biplane method (BIP) [2], are well established; they are somewhat time consuming, especially in the setting of an emergency "quick-look" situation. There is therefore much interest in developing a reliable method for the rapid assessment left ventricular ejection fraction (LVEF). We evaluated the performance of two transthoracic echocardiographybased methods of LVEF estimation in both experienced and novice readers with the objective of determining whether a simple semiquantitative method could improve LVEF estimation in both novice and experienced echocardiographers.

\section{Methods}

2.1. Study Setting. The study was conducted at a tertiary care academic teaching hospital in Montréal, QC, Canada. Data collection was conducted from July 2007 to February 2008. Most of the data was collected in small group sessions of approximately one hour in duration with between 5 and 7 readers at a time.

2.2. Participants. Five certified echocardiographers and 11 clinical echocardiography fellows and technicians comprised the expert image-reader group (PRO), and 105 medical students and physicians without any formal training in echocardiography comprised the novice group (NOV). 


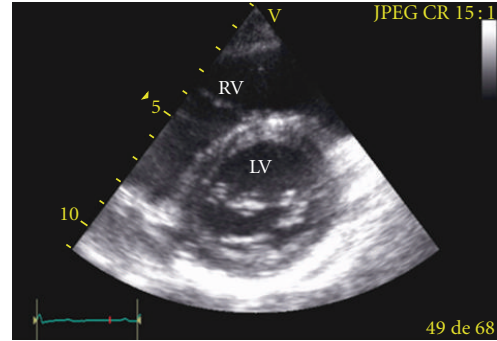

(a) Short-axis view of the left ventricular (LV) base

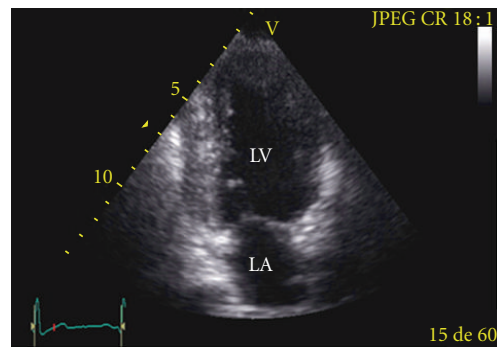

(d) Apical 2-chamber view

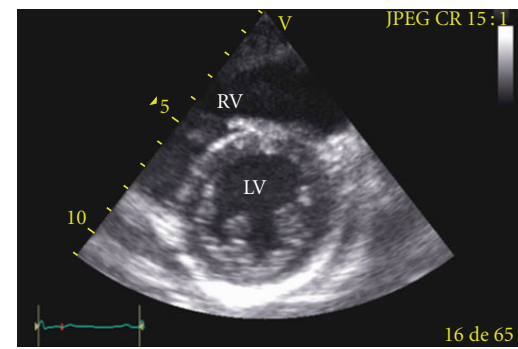

(b) Short-axis view of the mid-LV

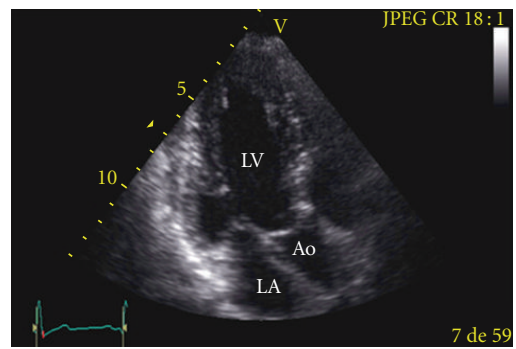

(e) Apical 3-chamber view

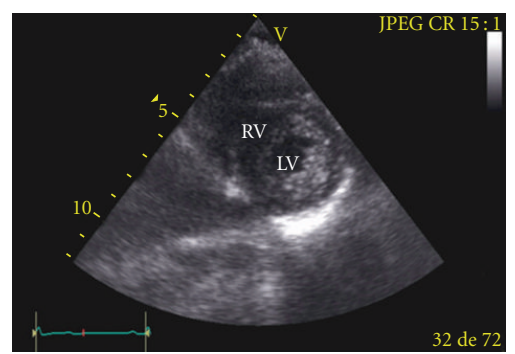

(c) Short-axis view of the LV apex

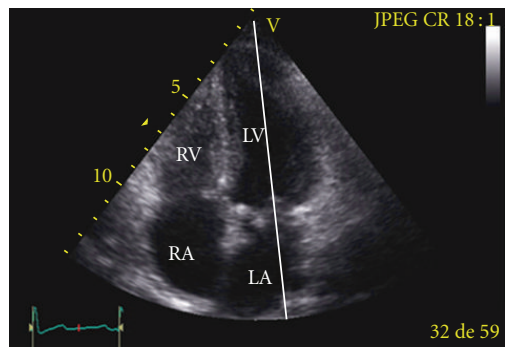

(f) Apical 4-chamber view

Figure 1: Standardized views of the BMA (a, b, c) and 234C (d, e, f) views.

2.3. Reference Imaging Data. Six representative cases were selected from a previous study [3]. These patients had LVEF established either by radionucleotide ventriculography (RNV) or by ultrasound using the biplane Simpson's method (BIP) [2]. Recorded-loop transthoracic images were obtained within three days of the LVEF evaluation using a Helwett-Packard Sonos 1500 or 5000 model ultrasound machine with a $2.5 \mathrm{MHz}$ transducer. Care was taken to ensure that the two-dimensional images displayed sufficient endocardial definition so as to allow for the unfettered evaluation of global and regional contractility of the left ventricle.

The six studies were comprised of two patients with low LVEF ( $\leq 30 \%)$, two patients with moderate LVEF (31\% to $49 \%)$, and two patients with normal LVEF ( $\geq 50 \%)$. Each of the six studies was comprised of three classic parasternal short axis views - the basal (B) or mitral valve level, the mid (M) or papillary muscle level, and the apical (A) level (Figures 1(a), 1(b), and 1(c), resp.)-and three classical apical views - the two- $(2 \mathrm{C})$, three- $(3 \mathrm{C})$, and four-chamber (4C) views (Figures $1(\mathrm{~d}), 1(\mathrm{e})$, and $1(\mathrm{f})$, resp.) a total of 36 clips. Both the BMA and $234 \mathrm{C}$ series of views were sufficient to allow determination of regional contractilities in each case.

\subsection{Study Protocol}

2.4.1. Global Visual Estimation (VIS). Readers were shown the six sets of images out of sequence and asked to evaluate the ejection fraction. They were asked to provide a global estimate of the LVEF based on a rapid appraisal of the three BMA and three $234 \mathrm{C}$ views, each shown for a mean of 15 cardiac cycles (minimum 10) in order to simulate quick-look conditions.
Prior to the test series, the echo-inexperienced group (NOV) was presented two brief sample cases: (i) a normal heart with normal segmental LV function in the three short axis and three apical views and (ii) an abnormal exam showing both hypokinesis (diminished movement and thickening of the LV wall segment) and akinesis (absence of movement and thickening of the LV wall segment). Participants were told that the normal exam represented an LVEF of $60 \%$. They were further told that an exam that was globally hypokinetic should be given an LVEF of $40 \%$ and one that was global akinetic $20 \%$. An exam presenting both normokinesis and hypokinesis would be ascribed a global LVEF of $50 \%$ and one that had mixed hypokinesis and akinesis $30 \%$. Similar instructions were given the participants in the echo-trained group (PRO). For example, their visual estimation was 50\%.

2.4.2. Semiquantitative Estimation (SQ). After the visual estimation of the LVEF of the six studies, the 105 NOV readers and 16 PRO readers received a brief explanation of the principle underlying the simplified WMSI-derived semiquantitative method [3]. The original WMSI used a 16-segment paradigm for the division of the $\mathrm{LV}$, as proposed by the ASE [4] (Figure 2). Each segment is given a score based on its systolic function (normal $=1$, hypokinesis $=2$, akinesis $=3$ ). The index (WMSI) is calculated by dividing the total of the wall motion scores of each segment by 16 . We have previously reported the linear regression model predicting RNV LVEF by WMSI [3] and several other studies have documented the good correlation of WMSI to LVEF [5-9].

2.4.3. BMA Series. Based on two published linear regression models $[3,9]$, it can be inferred that normal wall motion grossly corresponds to an LVEF of $60 \%$, mild hypokinesis 


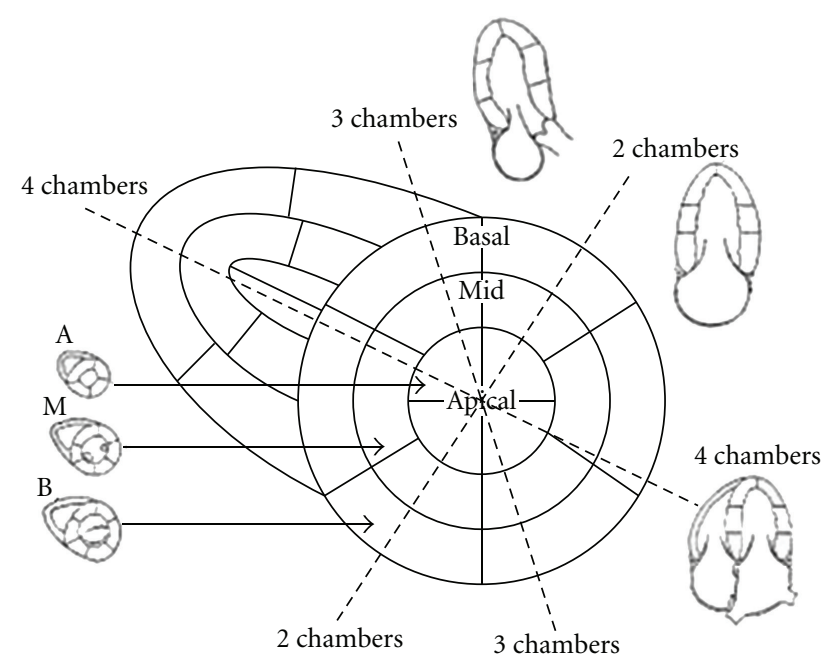

Figure 2: Sixteen-segment model as suggested by the American society of Echocardiography.

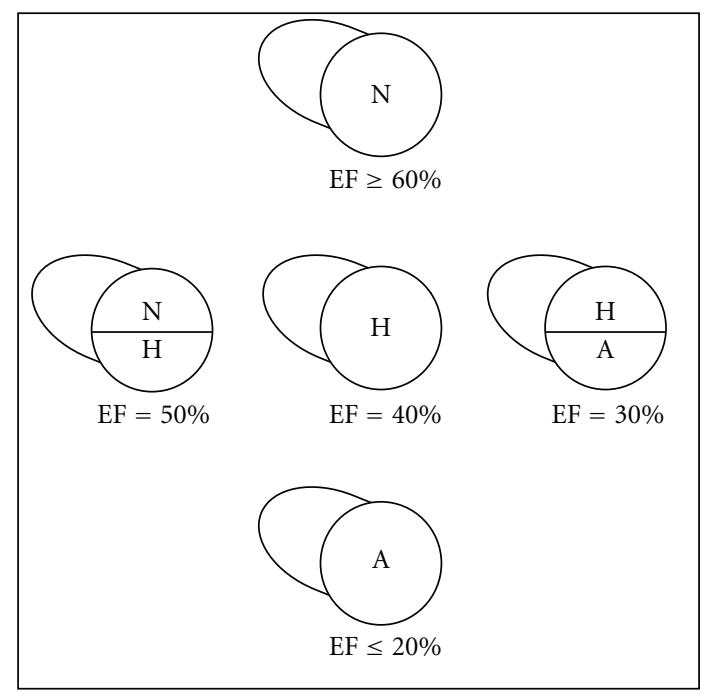

FIgURE 3: Schematic LVEF.

to $50 \%$, moderate hypokinesis to $40 \%$, severe hypokinesis to $30 \%$, and akinesis to $20 \%$ LVEF (Figure 3 ). Using this approximation, a simplified WMSI (sWMSI) was created such that readers would be asked to evaluate each image in the series and to provide a gradation of wall motion as either normal $(\mathrm{N})$, hypokinetic $(\mathrm{H})$, or akinetic $(\mathrm{A})$. Based on the reader's evaluation, the overall semiquantitative (SQ) LVEF was calculated for the BMA series by adding the inferred segmental LVEF's of each image and dividing by 3. For example, a BMA series evaluated as Base normal, Mid hypokinetic, and Apex akinetic would have a simplified WMSI LVEF of $(60 \%+40 \%+20 \%) / 3=40 \%$.

2.4.4. 234 C Series. For the series of apical views, the sWMSI was applied similarly to the method for the BMA series for the 105 participants in the NOV group.
2.5. Statistical Analysis. To assess agreement between each method of LVEF estimation and the gold standard (RNA or BIP), LVEF estimates and scores were transformed into 3 ordinal categories: severe depressed $(\leq 30 \%)$, mildly depressed (31-49\%), and normal ( $\geq 50 \%)$ LVEF. All analyses were performed separately for each patient and for each group (NOV and PRO). A concordance coefficient (between 0 and $100 \%$ ) was calculated, defined as the total number of agreement (LVEF in the same category by estimate and gold standard) divided by the total number of cases scored. Differences in the performance of LVEF estimation methods were evaluated using the chi-square method. Where a difference between the four estimation methods (VIS-BMA, VIS-234C, SQ-BMA, and SQ-234C) was observed, data was aggregated to compare the VIS and SQ methods, as well as the BMA and 234C series of views. The significance level for all analyses was set at 0.05 and performed with SPSS version 17.0 .

\section{Results}

All 16 members of the PRO group evaluated the six echocardiograms using both the VIS and SQ methods. Of those in the NOV group, 103 evaluated the six studies using the VIS method and 101 using the SQ method. The percent concordance of reader LVEF estimates with the reference standard are given in Table 1.

Experienced readers (PRO) fared equally well with either visual estimates or using the SQ method. NOV readers, on the other hand, performed better when using the VIS method for low LVEF situations, but with the SQ methods for normal or mildly depressed LVEFs.

Similarly, PRO readers performed equally well with either series of views. NOV readers had significantly better concordance with the reference standard in low LVEF situations using the $234 \mathrm{C}$ series of views. However, in estimating normal LVEFs, the BMA series resulted in better agreement. Either series of views resulted in similar agreement rates in the setting of mildly depressed LVEF.

\section{Discussion}

The accurate estimation of LVEF is central to the echographic assessment of patients in a multitude of settings. Increasingly, noncardiologists are called upon to rapidly evaluate echographic data, particularly in the emergency and critical care fields. It is therefore paramount to identify a reliable means of assessing LVEF for the noncardiologist. The principle finding of the present study is that a simplified wall-motion score index with relatively little instruction results in markedly improved LVEF estimates among echo-naïve readers.

The visual estimation of LVEF is widely used by experienced echocardiographers and has been shown to correlate well with the LVEF obtained from RNV $(r=0.89$ [10]; $r=0.91$ [11]). Akinboboye et al. [12] demonstrated that a good rate of correspondence using visual estimation can be obtained after only 60 evaluations if proper feedback is given to novice readers. Moreover, it has been suggested 
TABLE 1: Percent concordance between RNA/BIP and participant estimates for each method and for each patient.

\begin{tabular}{|c|c|c|c|c|c|c|}
\hline \multirow{2}{*}{$\begin{array}{l}\text { Type of observer } \\
\text { Patient }\end{array}$} & \multicolumn{2}{|c|}{ BMA } & \multicolumn{2}{|c|}{$234 \mathrm{C}$} & \multicolumn{2}{|c|}{ Sig best } \\
\hline & Visual & SQ & Visual & SQ & BMA/234C & Method \\
\hline Professional & $(N=16)$ & $(N=16)$ & $(N=16)$ & $(N=16)$ & & \\
\hline Patient $1(\mathrm{EF}=18 \%)$ & 100 & 100 & 100 & 100 & NS & NS \\
\hline Patient $2(\mathrm{EF}=29 \%)$ & 81.3 & 50.0 & 81.3 & 75.0 & NS & NS \\
\hline Patient $3(\mathrm{EF}=36 \%)$ & 81.3 & 93.8 & 75.0 & 75.0 & NS & NS \\
\hline Patient $4(\mathrm{EF}=40 \%)$ & 56.3 & 81.3 & 75.0 & 81.3 & NS & NS \\
\hline Patient $5(\mathrm{EF}=63 \%)$ & 94.8 & 94.8 & 87.5 & 81.3 & NS & NS \\
\hline Patient $6(\mathrm{EF}=72 \%)$ & 100 & 100 & 93.8 & 93.8 & NS & NS \\
\hline Novice & $(N=103)$ & $(N=101)$ & $(N=103)$ & $(N=101)$ & & \\
\hline Patient $1(\mathrm{EF}=18 \%)$ & 82.5 & 52.5 & 86.4 & 69.6 & $234 \mathrm{C}$ & Visual \\
\hline Patient $2(\mathrm{EF}=29 \%)$ & 20.4 & 4.0 & 93.3 & 60.2 & $234 \mathrm{C}$ & Visual \\
\hline Patient $3(\mathrm{EF}=36 \%)$ & 53.9 & 84.8 & 60.2 & 67.3 & NS & SQ \\
\hline Patient $4(\mathrm{EF}=40 \%)$ & 65.7 & 86.3 & 63.1 & 75.2 & NS & SQ \\
\hline Patient $5(\mathrm{EF}=63 \%)$ & 90.4 & 96.1 & 63.1 & 70.6 & BMA & NS \\
\hline Patient $6(\mathrm{EF}=72 \%)$ & 93.2 & 98.0 & 67.6 & 83.0 & BMA & SQ \\
\hline
\end{tabular}

Sig best: significant best method with a $P$ value $\leq 0.05$; NS: nonsignificant.

that teaching LVEF visual estimation by means of pattern recognition may be even more efficient in providing a rate correlation with RNV-derived measurements that approaches that experienced readers $(r=0.88)$ [13].

We have previously described usefulness of a semiquantitative wall-motion score index (WMSI) method of LVEF estimation for experienced echocardiographers [3], and a systematic review revealed that WMSI, Simpson biplane (BIP) and visual estimation by experience readers were nearly equivalent when RNV was used as a reference standard [14].

In the present study, a simplified version of the WMSI (sWMSI) demonstrated the capacity to improve the performance of novice-echocardiogram readers. Moreover, this improvement in the rate of correspondence with RNV was obtained with little prior instruction in the technique (only two example ultrasounds were provided to teach the scoring of the sWMSI.)

Certain limitations deserve mention here. First, although prospective in nature, the present study was not randomized. Second, although an attempt was made to recreate the conditions of an acute care scenario by limiting the views to an average of only 15 images, this may not have been sufficient to reproduce the stress of performing an examination in the setting of the emergency room or intensive care unit. As such, it may be that performance would not be as good under real life circumstances. On the other hand, readers in the real world may review images multiple times in order to form an opinion, which may compensate for any decreased performance due to stressful conditions. (Short viewing times were also felt to be essential in order to minimize any training effect.) Additionally, it should be noted that a number of wall motion abnormalities normally encountered in day-to-day practice were not evaluated in this analysis, such as hyperkinesia and dyskinesia and aneurysm and association of significant valvular disease (mitral insufficiency etc.). Finally, it is important to underline that the present study is one of interpretation of echo images and does not speak at all to the extensive training required for the proper acquisition of images [15]. The images used in this study were obtained by expert ultrasonographers, and the results of this study should in no way be interpreted to mean that the sWMSI could substitute for rigorous hands-on training in echo technique.

\section{Conclusion}

While visual estimation may be an adequate method to rapidly evaluate LVEF in experienced readers, the present study suggests that a semiquantitative method involving a simplified WMSI can improve LVEF estimates in noviceecho readers and can be used as a support in the training procedure. The present study, however, should not be interpreted to underscore the necessity of formalized rigorous training (three months) [15] in proper image acquisition technique and interpretation, within which the use of this simple, semiquantitative LVEF estimation method and appropriate feedback could be incorporated.

\section{Abbreviations}

LVEF: Left ventricular ejection fraction

BMA: Classical short axis of LV Base, Mid and Apex

234C: Classical apical view 2 chambers, 3 chambers, and four chambers

RNV: Radionucleotide ventriculography

BIP: Planimetric LV biplane Simpson's method

SQ: Semiquantitative evaluation of LVEF

VIS: Visual evaluation of LVEF

PRO: Experienced readers

NOV: Novice readers 
WMSI: Wall-motion score index

sWMSI: Simplified version of wall-motion score index.

\section{References}

[1] A. J. Labovitz, V. E. Noble, M. Bierig et al., "Focused cardiac ultrasound in the emergent setting: a consensus statement of the American society of echocardiography and American college of emergency physicians," Journal of the American Society of Echocardiography, vol. 23, no. 12, pp. 1225-1230, 2010.

[2] N. B. Schiller, H. Acquatella, T. A. Ports et al., "Left ventricular volume from paired biplane two-dimensional echocardiography," Circulation, vol. 60, no. 3, pp. 547-555, 1979.

[3] R. Lebeau, M. Di Lorenzo, R. Amyot, M. Veilleux, R. Lemieux, and C. Sauve, "A new tool for estimating left ventricular ejection fraction derived from wall motion score index," Canadian Journal of Cardiology, vol. 19, no. 4, pp. 397-404, 2003.

[4] N. B. Schiller, P. M. Shah, M. Crawford et al., "Recommendations for quantification of the left ventricle by two-dimensional echocardiography. American society of echocardiography committee on standards, subcommittee on quaititation of two-dimentional echocardiograms," Journal of the American Society of Echocardiography, vol. 2, no. 5, pp. 358367, 1989.

[5] R. D. Rifkin, H. Koito, M. Farmelant, and M. Skowronski, "Segmental model for estimating left ventricular ejection fraction by two-dimensional echocardiography: comparison with gated blood pool scanning," American Journal of Noninvasive Cardiology, vol. 4, no. 4, pp. 193-202, 1990.

[6] R. D. Rifkin and H. Koito, "Comparison with radionuclide angiography of two new geometric and four nongeometric models for echocardiographic estimation of left ventricular ejection fraction using segmental wall motion scoring," American Journal of Cardiology, vol. 65, no. 22, pp. 1485-1490, 1990.

[7] J. Berning, P. F. Hoilund-Carlsen, G. G. Nielsen, N. Gadsboll, J. Marving, and B. H. Jensen, "Critical reappraisal of bedside echocardiographic parameters for estimation of left ventricular ejection fraction in acute myocardial infarction. Importance of the wall motion index," American Journal of Noninvasive Cardiology, vol. 6, no. 5, pp. 269-278, 1992.

[8] J. Berning, J. R. Nielsen, J. Launbjerg, J. Fogh, H. Mickley, and P. E. Andersen, "Rapid estimation of left ventricular ejection fraction in acute myocardial infarction by echocardiographic wall motion analysis," Cardiology, vol. 80 , no. 3-4, pp. 257266, 1992.

[9] J. E. Møller, G. S. Hillis, J. K. Oh, G. S. Reeder, B. J. Gersh, and P. A. Pellikka, "Wall motion score index and ejection fraction for risk stratification after acute myocardial infarction," American Heart Journal, vol. 151, no. 2, pp. 419-425, 2006.

[10] R. B. Stamm, B. A. Carabello, D. L. Mayers, and R. P. Martin, "Two-dimensional echocardiographic measurement of left ventricular ejection fraction: prospective analysis of what constitutes an adequate determination," American Heart Journal, vol. 104, no. 1, pp. 136-144, 1982.

[11] A. F. Amico, G. S. Lichtenberg, S. A. Reisner, C. K. Stone, R. G. Schwartz, and R. S. Meltzer, "Superiority of visual versus computerized echocardiographic estimation of radionuclide left ventricular ejection fraction," American Heart Journal, vol. 118, no. 6, pp. 1259-1265, 1989.
[12] O. Akinboboye, J. Sumner, A. Gopal et al., "Visual estimation of ejection fraction by two-dimensional echocardiography: the learning curve," Clinical Cardiology, vol. 18, no. 12, pp. 726-729, 1995.

[13] M. D. Hope, E. de la Pena, P. C. Yang, D. H. Liang, M. V. McConnell, and D. N. Rosenthal, "A visual approach for the accurate determination of echocardiographic left Ventricular ejection fraction by medical students," Journal of the American Society of Echocardiography, vol. 16, no. 8, pp. 824-831, 2003.

[14] J. H. McGowan and J. G. F. Cleland, "Reliability of reporting left ventricular systolic function by echocardiography: a systematic review of 3 methods," American Heart Journal, vol. 146, no. 3, pp. 388-397, 2003.

[15] G. A. Beller, R. O. Bonow, and V. Fuster, "ACCF 2008 recommendations for training in adult cardiovascular medicine core cardiology training (COCATS 3) (revision of the 2002 COCATS training statement)," Journal of the American College of Cardiology, vol. 51, no. 3, pp. 335-338, 2008. 


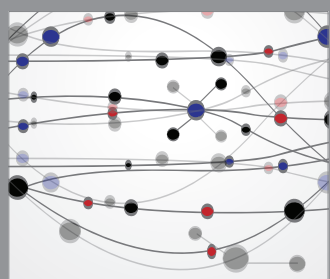

The Scientific World Journal
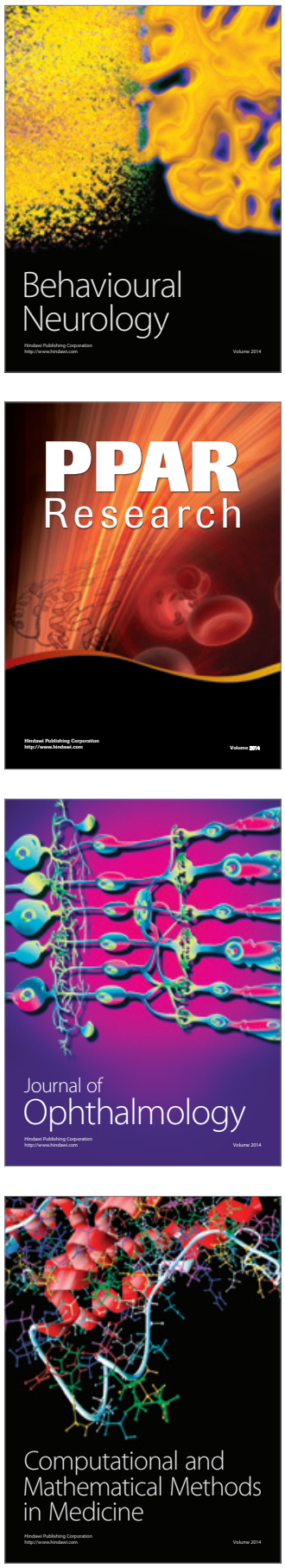

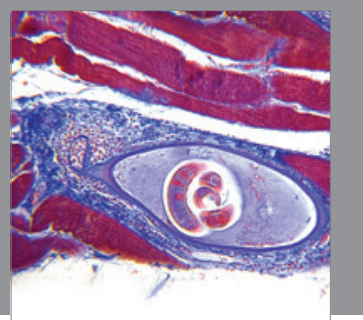

Gastroenterology

Research and Practice
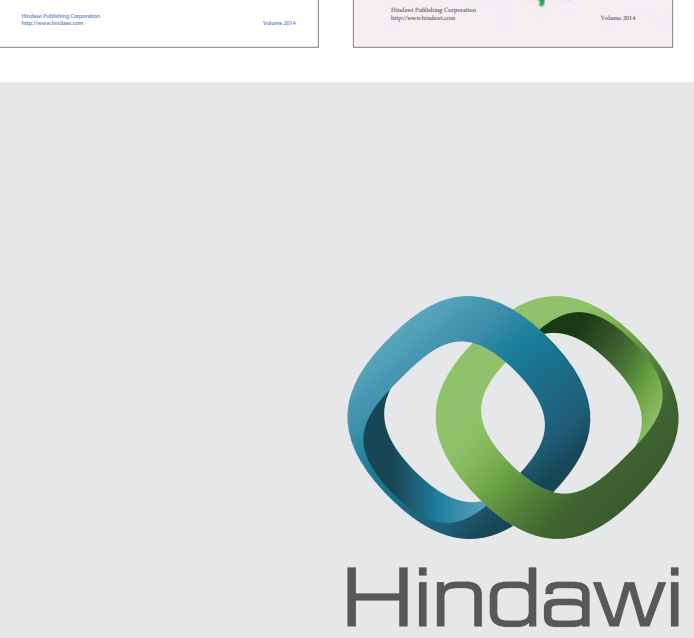

Submit your manuscripts at

http://www.hindawi.com
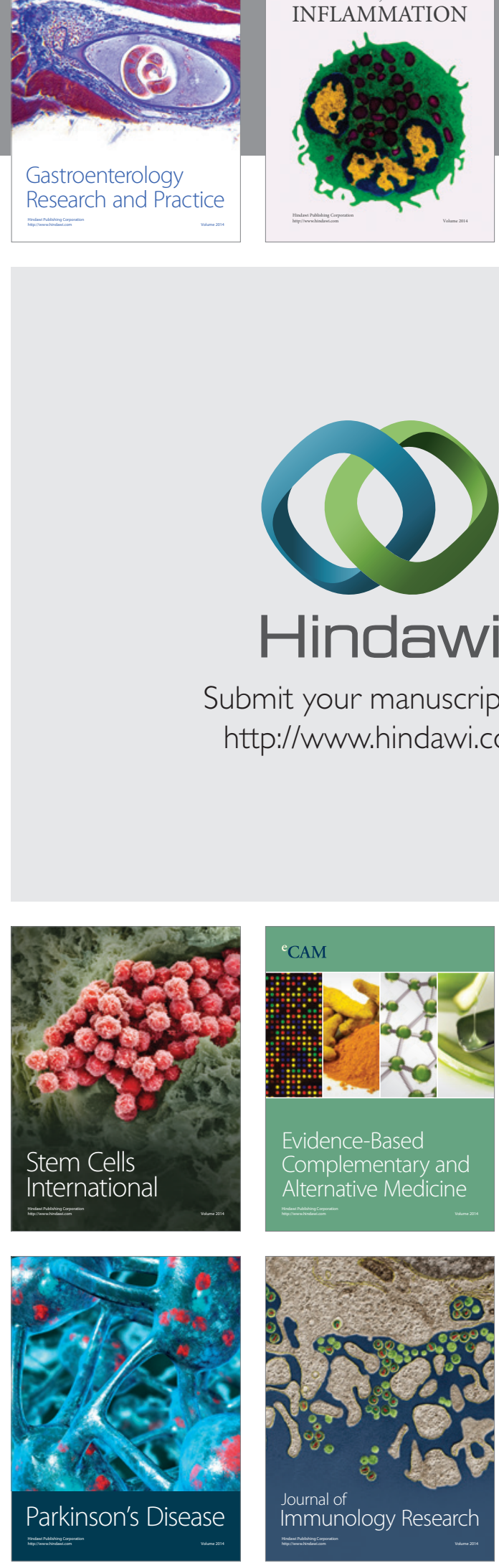

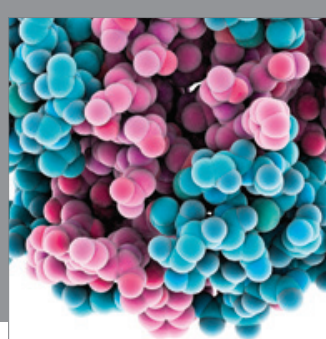

Diabetes Research
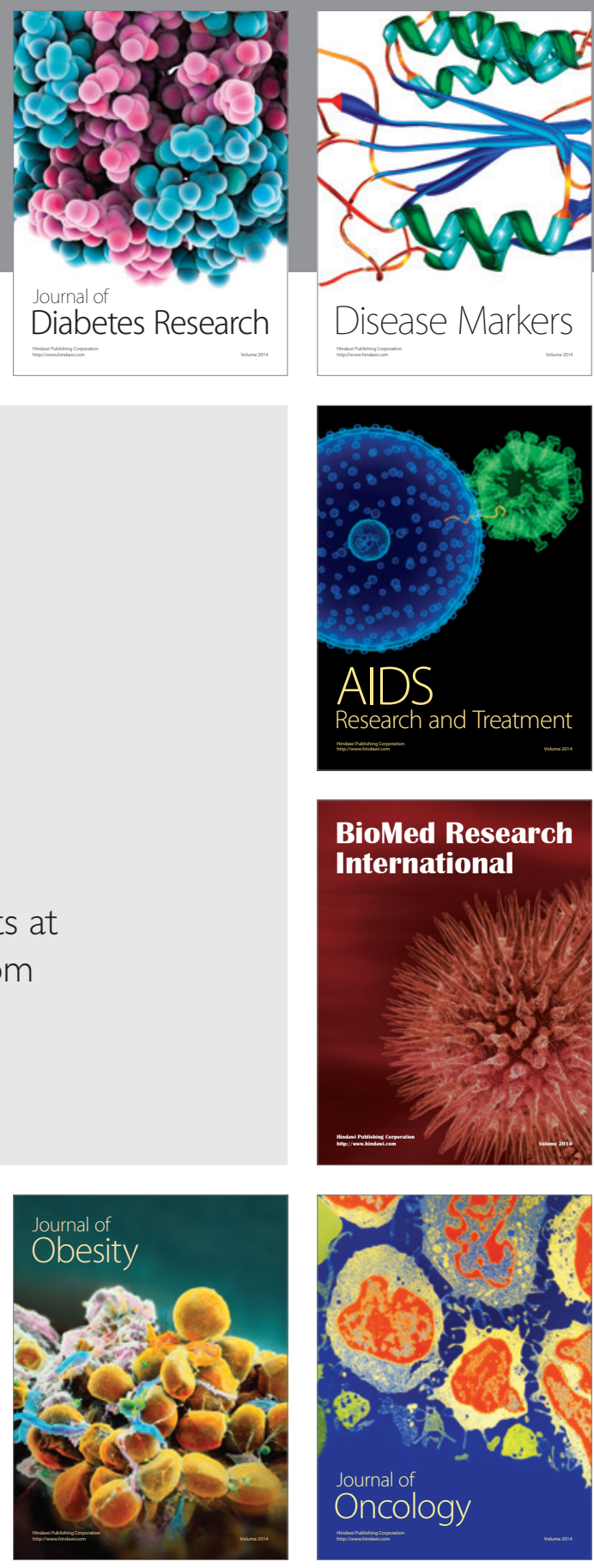

Disease Markers

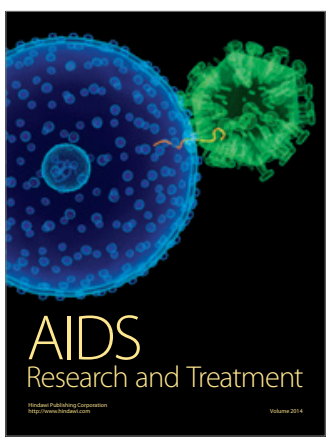

BioMed Research

International
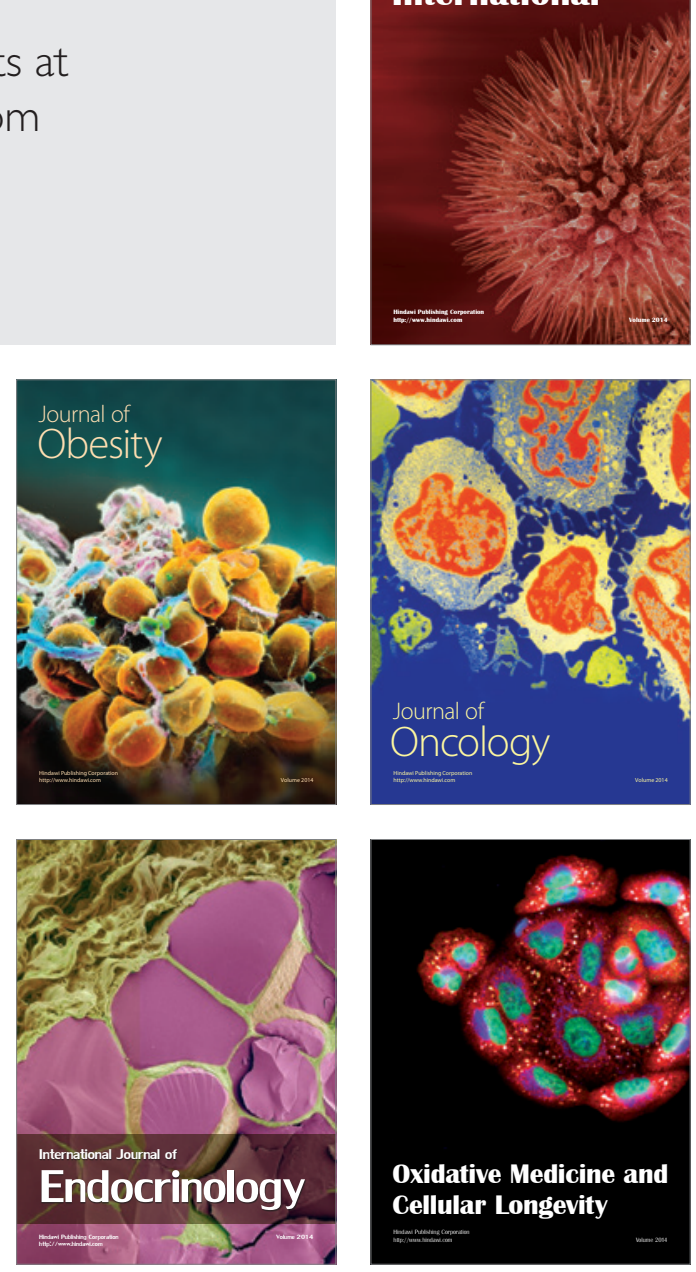